\title{
AN INTELLIGENT HYBRID CONTROL FOR PAPER MACHINE SYSTEM

\author{
G.Suribabu $^{1}$, T.Chiranjeevi ${ }^{2}$, T.Gurubhavan ${ }^{3}$, K. Ramesh kumar ${ }^{4}$ \\ ${ }^{1,2}$ Assistant Professor, EEE Department, Vishnu Institute of Technology, A.P, India, \\ ${ }^{3}$ Assistant Professor, EEE Department, Audisankara college of Engineering, A.P, India, \\ ${ }^{4}$ Project officer, EEE Department, IIT Hyderabad, A.P, India, \\ surigolla@yahoo.com,chirupci479@gmail.com,bhavaneee_217@yahoomail.com,ramesh@iith.ac.in
}

\begin{abstract}
The aim of this paper is to present an intelligent hybrid controller for a paper machine system with ash content and dry weight as outputs and filler valve and thick stock valve as inputs. Simulation studies have been carried out to check the robust performance (10\% increase in each process gain, 10\% increase in each time delay, and 10\% decrease in each time constant) of the system. The improvement in the performance of proposed hybrid controller is compared with the Adaptive-neuro-fuzzy controller and Dahlin controller and evaluated in terms of ISE.
\end{abstract}

Index Terms: Dahlin, Neuro-fuzzy, Hybrid and Robust

\section{INTRODUCTION}

Control system for paper machines is very complex as the overall system is complicated and is at least unknown. New control techniques for paper machines is usually evaluated in terms of its contribution to fibre efficiency, energy efficiency, reduced downtime and breaks increased product quality and increased machine capacity. In order to achieve these efficiency determinants of the paper machine system, ash content control plays a vital role [1].

An increased complexity is that ash content is also heavily coupled with another important variable, basis weight. Many control strategies have therefore been developed and applied to some production machines. However, most of these control algorithms [4] have assumed the linearity of the process, which is unlikely true for paper machines where frequent grade changes take place. It is therefore necessary to investigate the use of nonlinear control laws for such systems. Since neuro-fuzzy modeling techniques have been shown to be able to model the unknown nonlinear process to a prespecified accuracy, these control algorithms are well suited for the paper machine system. The later will then be combined with the dahlin controller to form an intelligent hybrid controller which outperforms the rest.

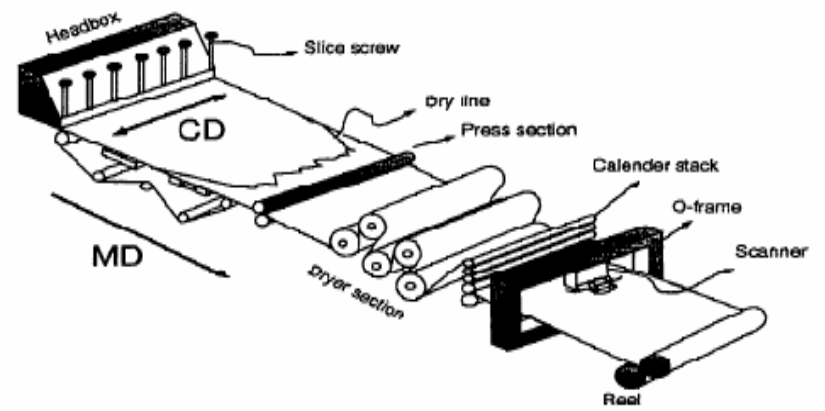

Fig 1.Paper Machine Unit

\section{PROCESS REPRESENTATION}

By denoting ash content in $(\%)$ and basis weight $(\mathrm{gm} / \mathrm{m} 2)$ as the outputs, filler flow rate and the thick stock rate as the inputs, the multivariable system of paper machine [2] is modeled as

$$
G(s)=\left[\begin{array}{ll}
G_{11}(s) & G_{12}(s) \\
G_{21}(s) & G_{22}(s)
\end{array}\right]
$$

Where

$$
\begin{array}{ll}
G_{11}=\frac{K_{a c}}{\tau_{a c} s+1} e^{-T_{a c} s} & G_{12}=\frac{K_{d a}}{\tau_{d w} s+1} e^{-T_{d w} s} \\
G_{21}=\frac{K_{a d}}{\tau_{a c} s+1} e^{-T_{a c} s} & G_{22}=\frac{K_{d w}}{\tau_{d w} s+1} e^{-T_{d w} s}
\end{array}
$$




$$
\begin{aligned}
& \text { Where } K_{a c}=0.214, \tau_{a c}=125, K_{d w}=0.93, \tau_{d w}=17 \\
& T_{a c}=T_{d w}=68, K_{a d}=0.153, K_{d a}=-0.192
\end{aligned}
$$

Which results the multivariable process as?

$$
G(s)=\left[\begin{array}{cc}
\frac{0.214}{125 s+1} e^{-68 s} & \frac{-0.192}{17 s+1} e^{-68 s} \\
\frac{0.153}{125 s+1} e^{-68 s} & \frac{0.93}{17 s+1} e^{-68 s}
\end{array}\right]
$$

This model is used in analysis of hybrid, neuro-fuzzy and conventional controller algorithms to track the desired basis weight and ash content properties in this paper.

\subsection{IMPLEMENTATION OF CONTROLLERS}

With the presence of strong interactions in the MIMO paper machine system ,the controller performance deteriorates and it is necessary to design a proper decoupler[3] in order to nullify the effect of interactions over the performance of controllers. Two important advantages of decoupling which were eliminated the interactions in paper machine system as follows:

1) Control loop interactions of ash content and basis weight were eliminated.

2) A set point change for ash content controlled variable not have any effect with basis weight controlled variable.

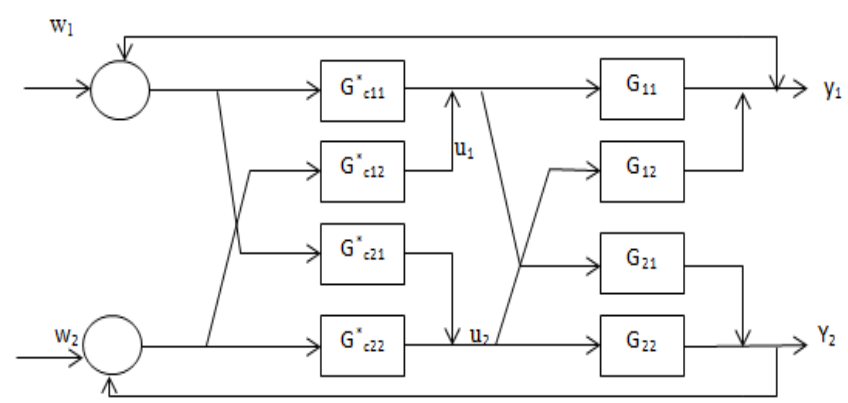

Fig 2.Decoupling Structure

If the transfer function elements of $\mathrm{G}$ are known and having specified the diagonal elements of $\mathrm{Gc}^{*}$, then the appropriate off-diagonal elements of $\mathrm{Gc}^{*}$ to achieve decoupling control can be calculated by using equations

$$
G_{c 12}^{*}=-\frac{G_{12} G_{c 22}^{*}}{G_{11}}
$$

$$
G_{c 21}^{*}=-\frac{G_{21} G_{c 11}^{*}}{G_{22}}
$$

\subsubsection{Dahlin and Adaptive Neuro-fuzzy controller}

A decoupler $\mathrm{D}(\mathrm{s})$ is included in the controller such that

$$
D(s) G(s)=\left[\begin{array}{cc}
G_{11}(s) & 0 \\
0 & G_{22}(s)
\end{array}\right]
$$

The choice of lambda as twice the original time constant [2]is a typical rule-of-thumb used in the paper mills while designing the Dahlin controller. In this paper the forward path controllers are replaced with neuro fuzzy controller to obtain better response.

As Fuzzy systems [5] lack the ability to learn and cannot adjust themselves to a new environment, the merger of a neural network with a fuzzy system into one integrated system, therefore, offers a promising approach in building intelligent systems. Integrated systems can combine the parallel computation and learning abilities of neural networks, with the human- knowledge representation and explanation abilities of fuzzy systems [6], [7].

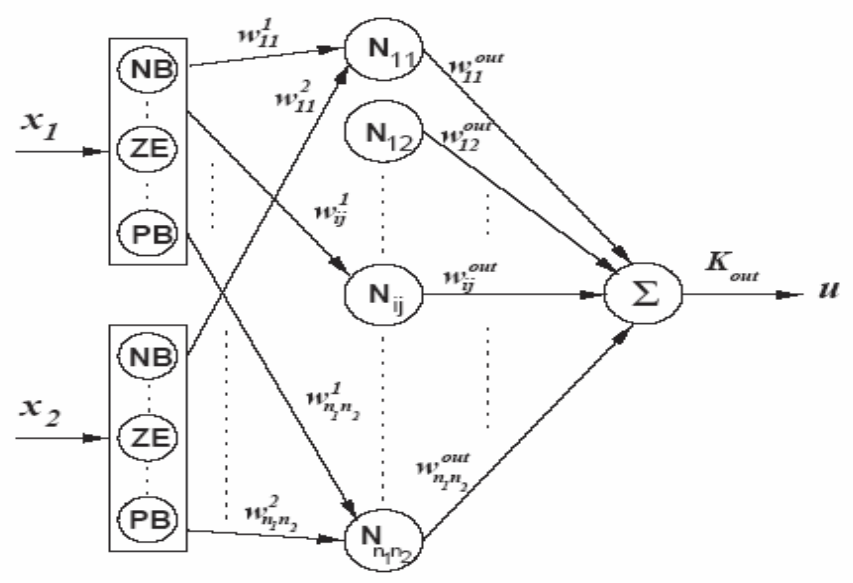

Fig3. Structure of the neuro-fuzzy control system

The Objective function to be minimized is

$$
E(k)=\frac{1}{2} \sum_{k}\left[y(k)-y_{r}\right]^{2}
$$

The learning rule could be expressed as

$u_{i j}(k+1)=u_{i j}(k)-\gamma_{b} \frac{\partial E(k)}{\partial u_{i j}(k)}$ 
$\begin{aligned} & \text { The gradient } \\ & \qquad u_{i j}(k)\end{aligned}=\frac{\mu_{i}\left(x_{1}(k), x_{2}(k)\right)}{\sum_{i=1}^{n} \mu_{i}\left(x_{1}(k), x_{2}(k)\right)} \psi_{1}$

Where $\psi_{1}=\sum_{k} J_{k} e(k)\left(w_{i}(k)-y(k)\right) \frac{1-\mu_{A i j}\left(x_{j}(k)\right)}{u_{i j}(k) \mu_{A i j}\left(x_{j}(k)\right)}$

$$
\begin{aligned}
& u_{i j}=\text { controller output of } \mathrm{jth} \text { input used in ith rule. } \\
& \gamma_{b}=\text { Learning coefficient. } \\
& \mu_{A i j}\left(x_{j}(k)\right)=\text { Degree of membership function } . \\
& w_{i}(k)=\text { Output of the ith rule } \\
& J_{k}=\text { Jacobian of the plant } \\
& \mathrm{e}(\mathrm{k})=\text { Error at the kth instant }=\mathrm{y}(\mathrm{k}) \mathrm{yr}
\end{aligned}
$$

The summation $\sum_{k}$ is for off-line learning that uses the summation of measurements at the discrete instances after the end of a control cycle. No summation $\sum_{k}$ is required in the above equations for on-line learning. In simulations, sgn of a discrete linearized Jacobian were used .Discrete linearized Jacobian is defined as

$J_{k}=\frac{y(k)-y(k-1)}{u(k)-u(k-1)}$

Its sgn defined by

$$
\operatorname{sgn}\left(J_{k}\right)= \begin{cases}1 & J_{k}>0, \\ 0 & \text { otherwise }\end{cases}
$$

\section{HYBRID CONTROLLER}

In this paper, a design methodology is introduced that blends the Dahlin and the fuzzy controllers in an intelligent way and thus a new intelligent hybrid controller[10],[11],[12] has been achieved. Basically, in this design methodology, the Dahlin and neuro-fuzzy controller have been combined by a blending mechanism that depends on a certain function of actuating error. Moreover, an intelligent switching scheme is induced on the blending mechanism that makes a decision upon the priority of the two controller parts. A switching \& blending mechanism firstly decides the dominant one of the two controller structures. The outputs of the neuro-fuzzy controller and the Dahlin controller are then multiplied by either one of the functions 1-f(e) and f(e).

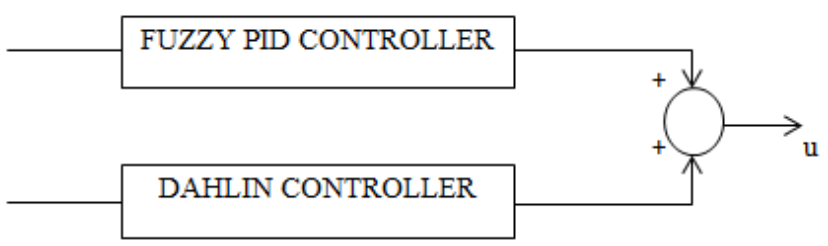

Fig 4: Structure of the hybrid control system

1-f(e) and $\mathrm{f}(\mathrm{e})$ are the weighing factors of the blending part of the mechanism. They quantify the level of the activity of the contributing controller and help us to achieve a reasonable tradeoff between the actions generated by the individual controllers. Since the function $\mathrm{f}(\mathrm{e})$ has to be positive valued, it has been selected as $\mathrm{f}(\mathrm{e})=\mathrm{e} 2$. Consequently the hybrid controller's output becomes either

$$
\text { UHYBRID }=f(e) \cdot U D+(1-f(e)) \cdot \text { UNFS }
$$

Or

$$
\text { UHYBRID }=(1-\mathrm{f}(\mathrm{e})) \cdot \mathrm{UPID}+\mathrm{f}(\mathrm{e}) \cdot \mathrm{UNFS}
$$

It is obvious that when the error is large the controller output multiplied by $\mathrm{f}(\mathrm{e})$ is activated more than the other controller part[13],[14]. For this reason, at the early stages of the control action, the controller output which gives the faster response must be multiplied by $f(e)$.

The switching part of the mechanism tries to catch the bigger one of the control efforts of the two main controller parts. The idea behind this is that higher control effort should produce faster system response. The simulations done on proposed system using the new hybrid fuzzy PID controller provides 'better' system responses in terms of transient and steady-state Performances when compared to the Dahlin and the neuro fuzzy controller.

\section{SIMULATION RESULTS}

Different intelligent control techniques were developed for paper machine control and simulated under Matlab 7.0 package to verify the performance of various control schemes with proposed hybrid controller. The feed forward decoupling elements replaced by PID controllers, designed using Dahlin method and the designed values for $G_{c 11}^{*}$ is kp=4.521, ,ki=0.1, $\mathrm{kd}=1$ and for ${ }_{c}^{*}{ }_{c}^{*} 22 \mathrm{kp}=0.1415, \mathrm{ki}=0.05, \mathrm{kd}=0.2$. 


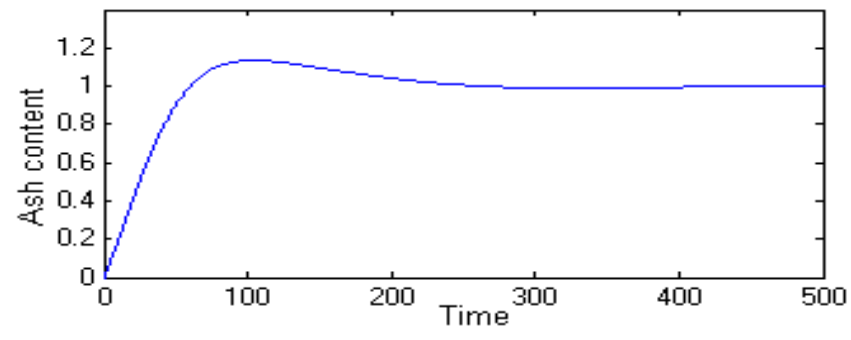

Fig5 Step response of ash content with Dahlin controller

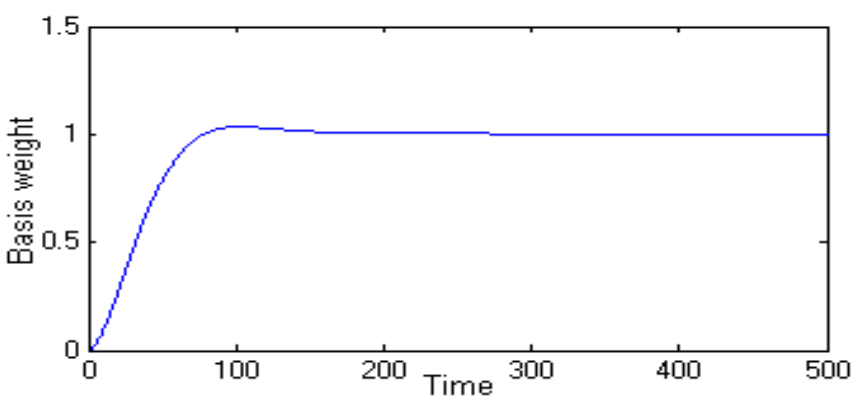

Fig6 Step response of basis weight with Dahlin controller

Neuro-Fuzzy logic controller is model free approach and it can deal effectively with parameter uncertainties. Therefore the two feed forward decupling elements are replaced with neurofuzzy controllers and simulated.

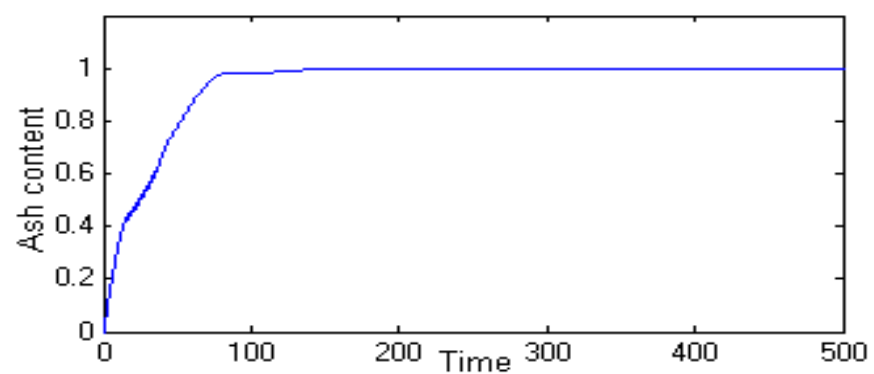

Fig7. Step response of ash content with neuro-fuzzy controller

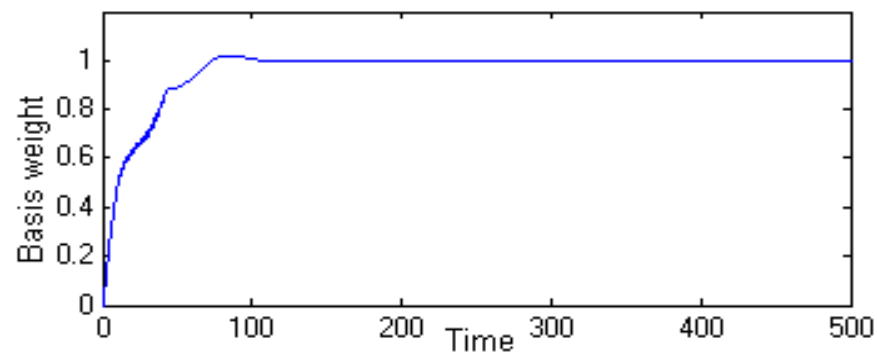

Fig8. Step response of basis content with neuro-fuzzy controller
From the simulation results (Fig.7,Fig.8), we can conclude that although neuro-fuzzy system is maintaining desired response but the response is not smooth initially, therefore we can overcome this problem by incorporating hybrid controller.

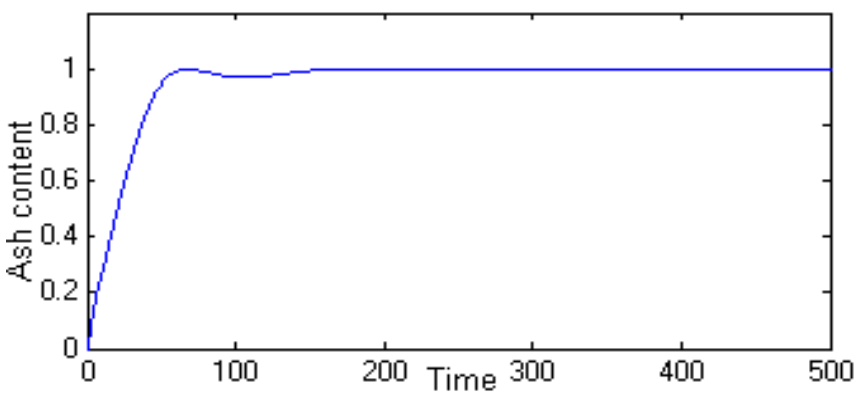

Fig9. Step response of ash content with hybrid controller

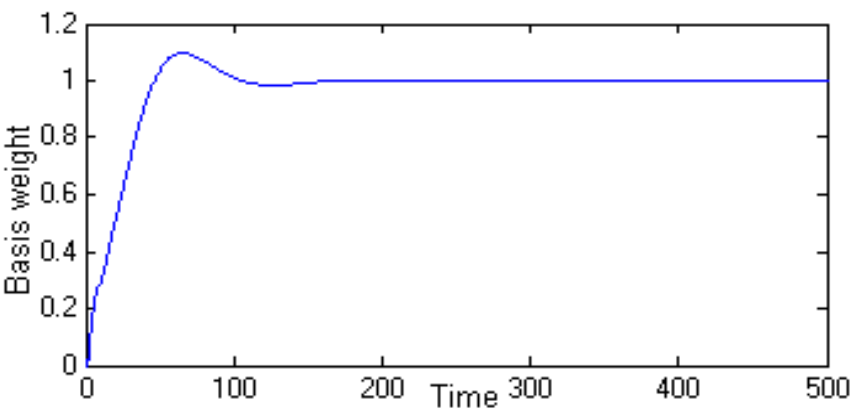

Fig10. Step response of basis weight with hybrid controller

Also to study the effectiveness of the hybrid controller, uncertainties in plant parameters were introduced by $10 \%$ increase in each process gain, $10 \%$ increase in each time delay, and $10 \%$ decrease in each time constant of the system to check robustness. The performance of hybrid controller was compared under disturbed plant parameters (Fig.12).

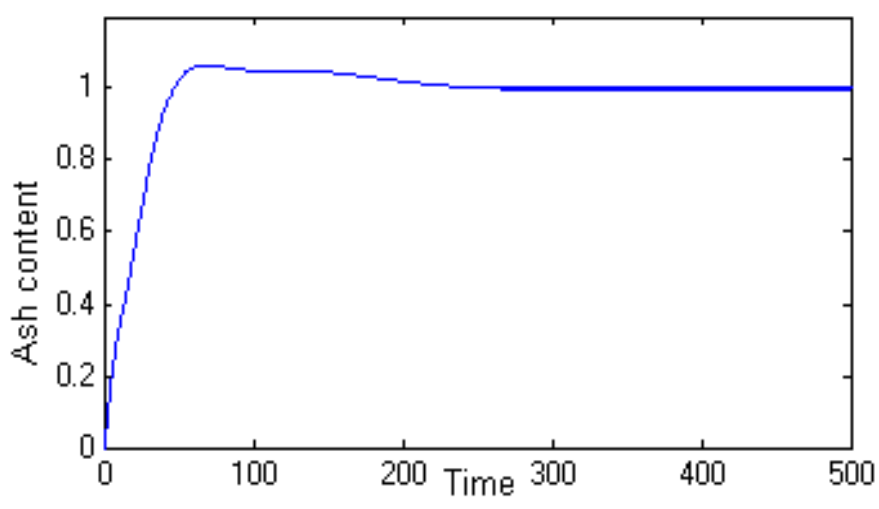




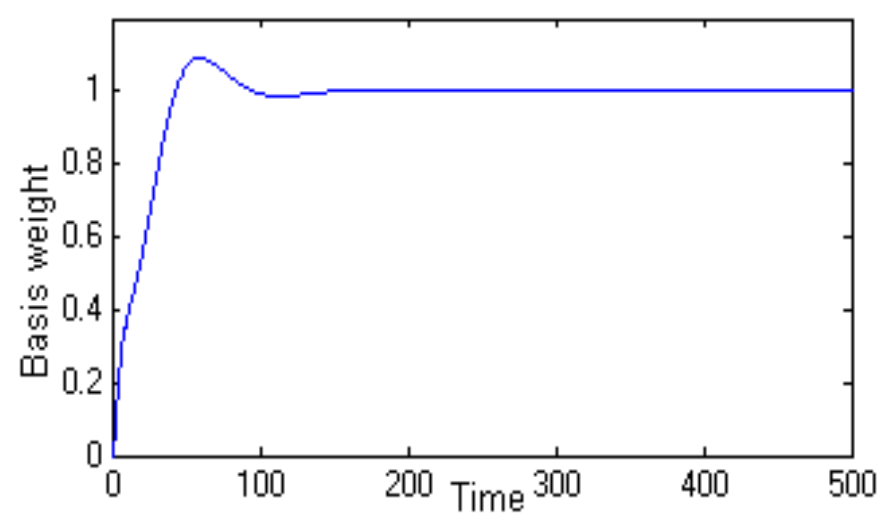

Fig11. Performance of the hybrid controller with disturbed plant parameters
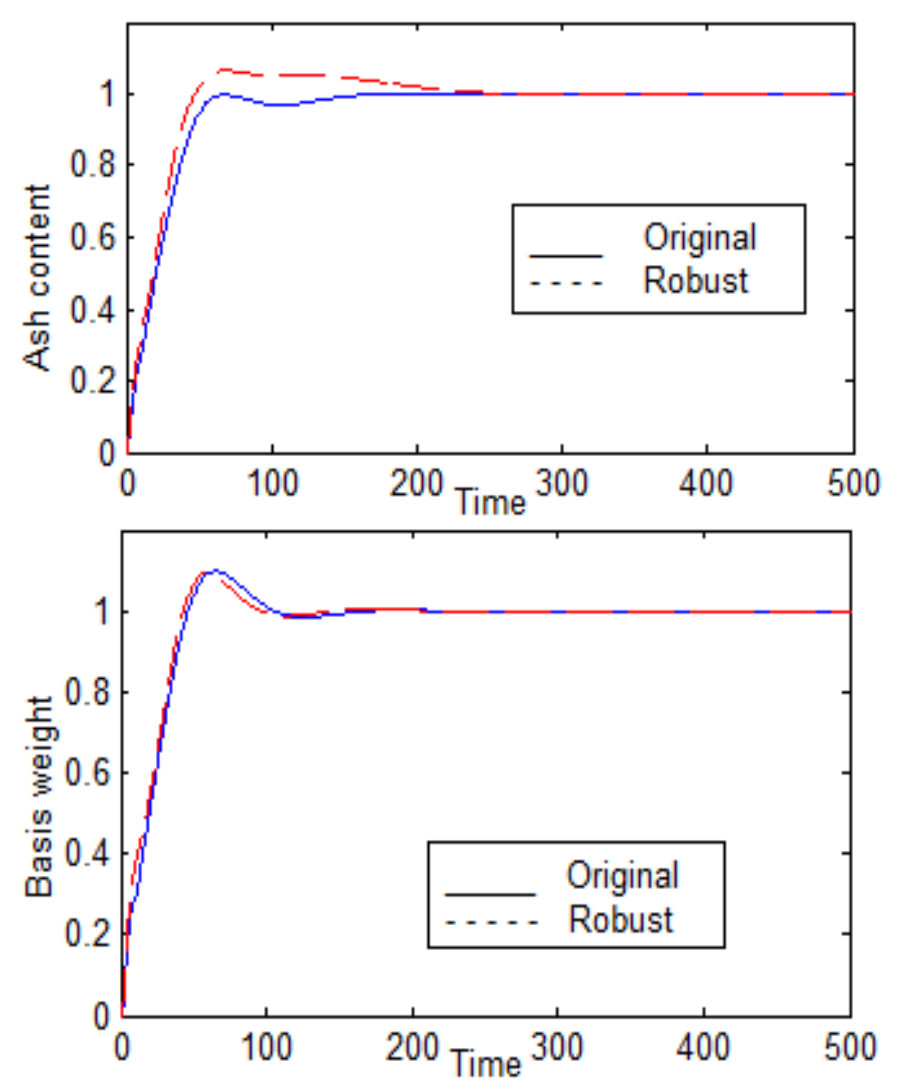

Fig12. Comparison of the performances of hybrid controller under disturbed plant parameters

From the fig (12) we can conclude that the proposed hybrid controller is robust. A comparison was made with the performances of Dahlin ,Neuro-fuzzy and hybrid controllers (Fig 13.).It is observed that the ISE values (Table.1) of proposed hybrid controller is reduced compared to the rest.
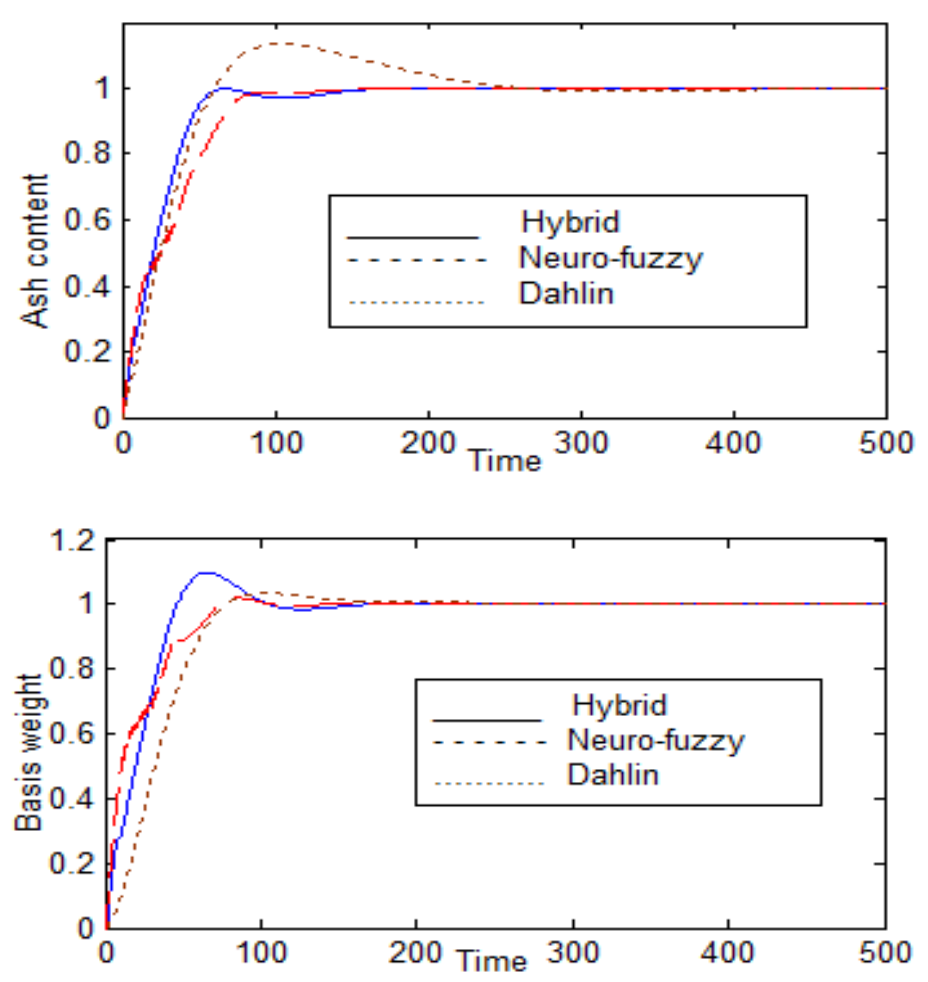

Fig13. Comparison of step responses of hybrid, neuro-fuzzy and dahlin controllers

Table -1: comparison of ISE values of Dahlin controller, Neuro-fuzzy controller and hybrid controller

\begin{tabular}{|l|l|l|}
\hline Controller & $\begin{array}{l}\text { ISE value for Ash } \\
\text { content }\end{array}$ & $\begin{array}{l}\text { ISE value for Basis } \\
\text { weight }\end{array}$ \\
\hline Dahlin & 9.746 & 12.62 \\
\hline Neuro-fuzzy & 8.382 & 7.057 \\
\hline Hybrid & 5.77 & 5.209 \\
\hline
\end{tabular}

\section{CONCLUSIONS}

From simulations it is concluded that, insufficient knowledge in making Knowledge base, forming rules and selection of membership functions effected Neuro-fuzzy controller performance.

The main shortcoming of Neuro-fuzzy system has also been tackled by integrating the Dahlin and neuro-fuzzy controllers. The simulation results presented in this paper has shown that the hybrid control can outperform the rest of the controllers in terms of robustness and parameter uncertainties.

A Comparison is made among all methods. ISE value are less in the case of hybrid control compared to the rest.(Table.1) . 


\section{REFERENCES}

[1]. Bergeron, M., Boulay, R., Drouin, B.,Gagnon, R., "Simultaneous Moisture and Basis Weight Measurement", Pulp and Paper Canada, Vol. 89, No. 12 pp. 173-176, 1988.

[2]Isaksson, A.J., L.E. Johnsson and M. Hagberg (1995). “ Benchmarking for Paper Machine MD-Control. Simulation Results", Extended Version. Technical Report TRITA-REG9502. Department of Signals, Sensors and Systems, Royal Institute of Technology. Stockholm, Sweden.

[3]M.T. Tham, "Multivariable Control an Introduction To Decoupling Control" , Dept. of Chemical and Process Engineering , University of Newcastle upon Tyne An introduction to Decoupling Control/MTT/July 1999.

[4] K.L. Tang and R.J. Mulholland, "Comparing fuzzy logic with classical controller designs", 1EEE Trans. Systems Man Cybernet. 17 (1987) 1085- 1087.

[5] Verbruggen, H.B. and Brujin, P.M."Fuzzy Control and Conventional Control - What is the real contributionof Fuzzy Systems"Fuzzy Sets and Systems 1997 (151-160).

[6]Braae, M., Rutherford, D.A., "Selection of Parameters for a Fuzzy Logic Controller", FuzzySets and Systems, Vol. 2, pp. 185-199, 1979.

[7] T. Takagi and M. Sugeno, "Fuzzy identification of systems and its applications to modeling and control," IEEE Trans. Sys ., man, Cybern, vol.SMC-15, pp. 116-132, 1985.

[8]Lee, C.C., "Fuzzy Logic in Control Systems: Fuzzy Logic Controller, part I", IEEE Transactions on Systems, Man and Cybernetics, Vol 20, Part I: pp. 404-418, Part II: pp. 419-435, 1990.

[9]C.C.Lee, "Fuzzy logic in control systems: fuzzy logic controller, Part II", IEEE Trans. Syst., man, Cybern, vol.SMC20. pp. 419-435, 1990.

[10] Brehm, T., Rattan, K.S., 1993. "Hybrid fuzzy logic PID controller." Proceeding of the IEEE National Aerospace and Electronics Conference, Vol:2, 807-813.

[11]Er, M. J., Sun, Y.L., 2001. "Hybrid fuzzy proportional integral plus conventional derivative control of linear and nonlinear systems." IEEE Trans.On Industrial Electronics 48(6),1109-1117.

[12] Li, W. 1998. "Design of hybrid fuzzy logic proportional plus conventional integral-derivative controller." IEEE Trans. Fuzzy Systems 6(4), 449-463.

[13] Li, W., Chang, X.G., Wahl, F.M., Tso, S.K., 1999. Hybrid fuzzy P+ID control of manipulators under uncertainty." Mechatronics (9), 301-315. 